\section{Targeting active cancer cells with smart bullets}

Paul Ehrlich's 'magic bullet' concept has stimulated research for therapeutic agents with the capability to go straight to their intended targets. The 'magic bullet' concept is still considered the ultimate approach to maximize the therapeutic effects of a given therapeutic agent without affecting nontargeted tissues. But so far, there has never been a therapeutic agent or a delivery system that goes straight to the target in the body, and no approach has provided anything better than just a few percents of the total administered dose reaching the intended target sites. But engineering principles can transform systematically circulating vectors that so far were based primarily on physical characteristics and biochemical principles alone, as smart therapeutic agents with the required propulsion-navigation-homing capabilities to enable them to go straight to their intended targets.

First draft submitted: 13 December 2016; Accepted for publication: 22 February 2017; Published online: 31 March 2017

Keywords: cancer translational research • clinical MRI scanner $\bullet$ high therapeutic index - magnetic fields $\bullet$ magnetotactic bacteria $\bullet$ nonsystemic therapeutic delivery $\bullet$ robotic functionalities $\bullet$ smart delivery agents $\bullet$ targeting active cancer cells $\bullet$ tumor hypoxic zones - vascular navigation

By analogy to modern warfare where smart bombs developed primarily by engineers replaced payloads delivered through carpet or saturation bombing, a given improvement in accuracy of a therapeutic delivery agent resulting in a reduction of the miss distance from active tumor cells would significantly enhance the therapeutic efficacy using fewer agents while causing much less systemic toxicity (collateral damages). If targeting effectively all active cancer cells in the primary tumor could be done at a sufficient early stage, then the risk of metastasis could be eliminated or at least reduced significantly with minimum invasiveness during the interventions. To do just that, each delivery vector would have to be sufficiently small while embedding the essential robotic functionalities to effectively target these tumor-active cells. As smart bombs or smaller version smart bullets, engineering principles can be used to transform therapeutic agents closer to the 'magic bullet' concept [1] when being applied to spatially localized targets by implementing fundamental functionalities such as propulsion, navigation and homing capabilities.

For instance, sufficient propelling force would be required for not only allowing these therapeutic agents to travel directly from the injection site to the tumor, but also to allow them to go deeper in tumoral tissue and toward regions of active cancer cells that are most often well beyond the diffusion limit of existing therapeutic vectors. Such diffusion limit is due mainly to the absence of flow in the tumoral tissue caused by the tumor interstitial fluid pressure [2].

To achieve nonsystemic delivery in order to eliminate or at least minimize systemic
Sylvain Martel

NanoRobotics Laboratory, Department of Computer \& Software Engineering, Institute of Biomedical Engineering, Polytechnique Montréal, Montréal, QC, Canada

sylvain.martel@polymtl.ca 
toxicity while increasing the therapeutic index, embedding navigation capability in each targeting agent becomes essential. Although larger blood vessels such as arteries can be visualized using existing medical imaging modalities, their spatial resolution is insufficient to gather information about potential pathways in the microvasculature and in interstitial microenvironments leading to regions of active tumor cells. Because of such a limitation, closed-loop displacement control [3] from a computer outside the patient is not an option. Therefore, each delivery agent must have sufficient autonomy to be able to find these physiological microscopic pathways while being directionally guided toward the tumor from an external source.

To maximize the therapeutic effects while eliminating or at least minimizing the risk of metastasis, it becomes essential to be able to target all active tumor cells with a sufficient number of delivery agents at an appropriate early stage. As such, besides early tumor detection, appropriate homing capability must be embedded in each delivery agent to effectively and autonomously seek regions of active tumor cells. One potential homing source that is common to regions of fast duplicating cancer cells in the tumor is a lower oxygen concentration. When a sufficiently large number of tumor cells duplicate at a rapid pace, they consume oxygen faster than the supply of oxygen, hence, creating what is well known as tumor hypoxic regions [4]. Targeting these hypoxic regions is therefore crucial since they are associated with diminished therapeutic responses and malignant progression, in other words, an increasing probability of recurrence and distant metastasis [5].

\section{Implementation}

The present implementation proposes a different approach from the systemic molecular-based delivery methods that have been investigated so far. It is focused on engineering aspects to achieve nonsystemic targeting and therapeutic delivery into a tumor area. It does not replace but instead complement what have been done so far in previous systemic-based delivery methods. As such, the following specifically describes these engineering supplements to help molecular structures and functionalities to improve the targeting ratio to active cancer cells. As such, no emphasis is placed here on molecular structures and recognition including passive and active targeting since they are already widely described in the literature and which remain valid in the context of this nonsystemic delivery approach. In other words, it is proposed here that engineering principles can be applied to help bringing and maintaining these molecular constructs within constrained physiological regions where they would lead to more effective treatments.
Adding the essential robotic functionalities to improve the targeting efficacy of existing molecular constructs is not an easy task since the potential delivery routes to reach these hypoxic regions limit the overall diameter of these 'smart' nonsystemic delivery agents to below approximately $2 \mu \mathrm{m}$. These routes include the irregular and disorganized angiogenic network, the interstitial spaces and the intercellular openings of less than $2 \mu \mathrm{m}$ between endothelial cells within solid tumors [6].

It is obvious that embedding all the fundamental robotic functionalities required to effectively target active cancer cells in a synthetic or artificial version at such a small scale is well beyond present technological feasibility. Furthermore, even if it would become possible in a relatively far future, the implementation cost per unit combined with the large number of these smart delivery agents estimated to be in the order of hundreds of millions per injection depending on the efficacy of the therapeutic payloads and the characteristics of the tumor, which would be required to deliver sufficient therapeutic doses, could be a major obstacle toward its adoption for clinical uses. As such, harnessing a natural agent in the form of a self-duplicating microorganism having the proper characteristics and robotic functionalities could prove to be a valid option at least in the shorter term, to implement at a relatively low cost such smart delivery agents [7].

One example is the use of magnetotactic bacteria [8] and more specifically the Magnetococcus marinus strain MC-1 cells [9] that, when operating in an artificially generated magnetic environment, can behave as 'smart' delivery agents (Figure 1) with the robotic functionalities required to deliver nonsystematically therapeutic payloads to the active tumor cells [10]. Indeed, the homing behavior of the MC-1 cells is provided by their onboard autonomous aerotactic capability [11] to target sources such as oxygen-depleted hypoxic regions. Since the distance from the site of injection to these targets far exceeds the homing range corresponding to the oxygen diffusion distance $(\sim 100 \mu \mathrm{m})$ in the tumoral volume [12], propulsion and navigational guidance toward the tumor followed by a searching phase within the tumoral volume are initially performed in order to reach the $\mathrm{O}_{2}$ diffusion distance required to trigger the homing process to target the hypoxic areas. In the case of the MC-1 cells, directional propelling force is provided by two bundles of flagella connected to rotary molecular motors embedded in each bacterial cell. A chain of intracellular iron-oxide $\left(\mathrm{Fe}_{3} \mathrm{O}_{4}\right)$ nanoparticles, known as magnetosomes [13] and synthesized in the cell during cultivation, allows magnetotactic navigational guidance from a directional magnetic field slightly higher than 
Figure 1. Photograph of the unloaded MC-1 bacterium (top left), with the therapeutic payloads in this particular example being in the form of 70 drug-loaded liposomes (diameter $170 \mathrm{~nm}$ ) attached to the MC-1 cell (diameter 1-2 $\mu \mathrm{m}$ ) (top right). In the lower photograph, the MC-1 bacterium is depicted with a superimposed simplified block diagram representing the main functional systems used to allow effective targeting of active cancer cells. In an engineering point of view, each MC-1 bacterium can be represented as a natural nanorobot carrying therapeutic payloads. A theoretical futuristic artificial equivalent of this 'natural nanorobot' would be represented with an onboard computer (A) that would be used to determine the appropriate actions from signals received from various sensors. An onboard navigation system (B) would be implemented to receive information from a special external system in our case being known as the magnetotaxis platform transmitting or indicating the direction of the tumor. During travel, an obstacle detector (C) would send signals to the onboard computer (A) that, in turn, would control each molecular rotary motor (E) connected to a flagellated propulsion system in order to move around physiological obstacles while maintaining its general orientation indicated by the navigation system (B). Once in the tumor, no guiding information would be transmitted by the magnetotaxis platform to the navigation system (B), and only the signals from the onboard oxygen sensors (D) would be taken into consideration to autonomously control the displacement of the microscopic robot with its obstacle detector (C) still active. The onboard computer (A) would then control each molecular rotary motor $(E)$ in order to move in the direction from high oxygen toward low oxygen concentrations. When the signals received from the oxygen sensors indicating that the oxygen concentration would be at the level expected in regions of active tumor cells $\left(\sim 0.5 \% \mathrm{O}_{2}\right)$, the computer $(\mathrm{A})$ would send commands to the rotary motors $(E)$ to reverse the sense of rotation of the flagellated propulsion systems. Forward and backward motions would follow by switching the sense of rotation of the molecular motors based on the level of oxygen being detected in order to maintain its position within the tumor hypoxic regions until the release of the therapeutic cargos. Finally, a power source (F) would provide the necessary power to maintain these robotic functions operational for a period of time sufficient to reach the active tumor cells. Although this sounds like science fiction, it describes the functions of the live MC-1 bacteria when operating under the computer-controlled magnetic environment of the magnetotaxis interventional platform to target active cancer cells.

the geomagnetic field pointing toward the tumoral volume. When in the tumoral volume delimitating the search area for potential hypoxic zones, magnetotactic navigational guidance is turned off or the corresponding directional magnetic field is set at a sufficiently low level to allow aerotactic displacements of the bacteria to take effect. Unless the MC-1 cells are already within the $\mathrm{O}_{2}$ diffusion range from the hypoxic regions, displacements following a randomized search pattern within the physiological spaces
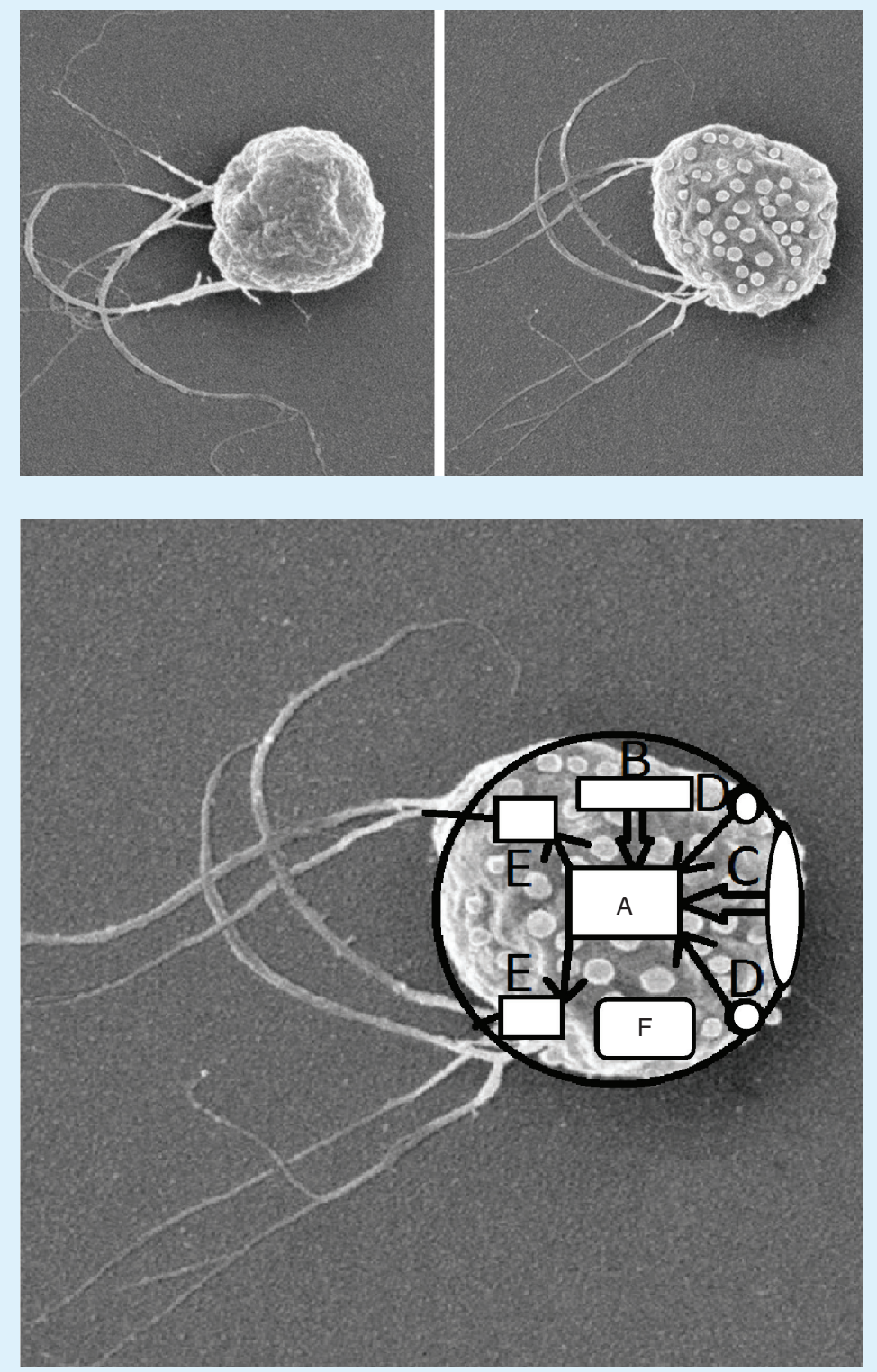

due to a lack of a directional magnetic torque on the chain of magnetosomes are autonomously initiated by the bacteria and continue until the MC-1 cells come within homing distance from the hypoxic zones. When within such homing range, the direction of the aerotactic displacement follows the decreasing oxygen gradients until the MC-1 homing $\mathrm{O}_{2}$ level of approximately $0.5 \%$ [11] corresponding to the microaerophilic threshold of the bacteria and which is equivalent to the oxygen concentrations at the targeted hypoxic 
areas [14] is reached. This specific oxygen threshold allows therapeutic-loaded MC-1 bacteria to maintain their position in hypoxic areas until the release of the therapeutic cargos. A simple schematic of the targeting procedure from an intra-arterial injection or a peritumoral injection is depicted in Figure 2.

\section{Therapeutic payloads}

Theoretically, any types of therapeutic payloads can be self-attached to the surface of the bacterial cell intended for spatially targeted chemotherapy, radiotherapy, immunotherapy, hyperthermia or stem cells delivery, to name but a few examples. These therapeutic payloads can be encapsulated in liposomes or a biodegradable polymer, to name but only two typical examples. The photograph in Figure 1 depicts one example showing approximately 70-170 nm liposomes, each containing SN-38 therapeutic molecules for the treatment of colorectal tumors. In this example, the drug-loaded liposomes were attached to the surface of the MC-1 cells using carbodiimide chemistry [15]. Knowing the volume and the number of therapeutic payloads being attached per cell, as demonstrated in the previous example, is important when the number of bacteria to be injected must be determined for a given dose and targeting ratio. Imaging, diagnostic or theranostic vectors could also be attached and transported by the same bacteria, providing valuable information to the clinicians including, but not limited to, targeting assessments, tracking tumor progression and the efficacy of the treatment, while extending further the types of applications that could be envisioned.

\section{Peritumoral interventional procedure}

So far, these nonsystemic delivery methods have been only tested on animals. But following very encouraging results, a translational phase aimed at developing appropriate medical protocols initially on larger animals has been initiated. To do just that, a special interventional suite has been put in place and will be upgraded based on observations during the interventions on larger animals prior to be considered for clinical uses in order to provide the most efficient combination of interventional platforms for each type of cancers.

Although several configurations of interventional suites ranging through various levels of complexity and types of interventions could have been implemented, the one that has been put in place to provide a suitable interventional facility for maximum targeting accuracy while supporting a relatively large range of possible therapeutic modalities and target physiological sites is depicted in Figure 3.
In this type of interventional room, the simplest interventional procedure occurs when the injection can be done in the periphery of the tumor, being referred to as peritumoral injection. Colorectal and prostate cancers are only two among several types of cancer where peritumoral injections can be performed. Based on last experiments, $55 \%$ of peritumorally injected drugloaded MC-1 cells reached the hypoxic zones in tumor xenografts implanted in mice compared with $1-2 \%$ with previous delivery methods [10]. Although similar targeting results are expected for humans, the interventional procedures that will be used will differ widely from the one that was used for the mice experiments.

Although various interventional procedures are possible, a standard and logical scenario that will initially be investigated would have, as a first step, the identification of the spatial location of the tumor or the area of potential regions where the cancer cells are likely to spread in the case of metastatic cancers. As an example for the latter case, it is well known that most of the time, prostate cancer cells metastasize to hip, spine and pelvis bones, hence, providing a spatially constrained target region for such nonsystemic delivery approach. A simple x-ray system or a more sophisticated CT scanner with a clinical MRI scanner (Figure 3) are likely to be used to identify and locate such spatially target regions. In most cases, the patient lying flat on a robotic table would typically be first robotically moved inside the tunnel of the MRI scanner. This would be initiated from the computer interfaces in the control room (Figure 3) where a medical specialist would be able to observe the scene through a radiofrequency (RF)-shielded window. Once the patient would be positioned, an MRI scan would be initiated from the computer interfaces.

Once the image of the tumor would be gathered and displayed on one of the computer screens, the operator or the medical specialist would then set the targeted volume using special functions provided by the computer interfaces. This targeted volume superimposed on the image of the tumor could be chosen to be of any sizes within a possible specific range of a special interventional platform dubbed the magnetotaxis platform (Figure 3). Such targeted volume would correspond to the volume delimitating the aerotactic search areas to target the hypoxic zones. For instance, the targeted volume could correspond to regions where cancer cells were detected or were expected to spread in the case of metastatic cancers, a whole tumor or a particular volume within a tumor. Once the targeted volume would be set on one of the computer displays, the robotic transfer of the patient from the MRI scanner to the magnetotaxis platform would be initiated from the control room which could be done with a simple click of the computer mouse. 


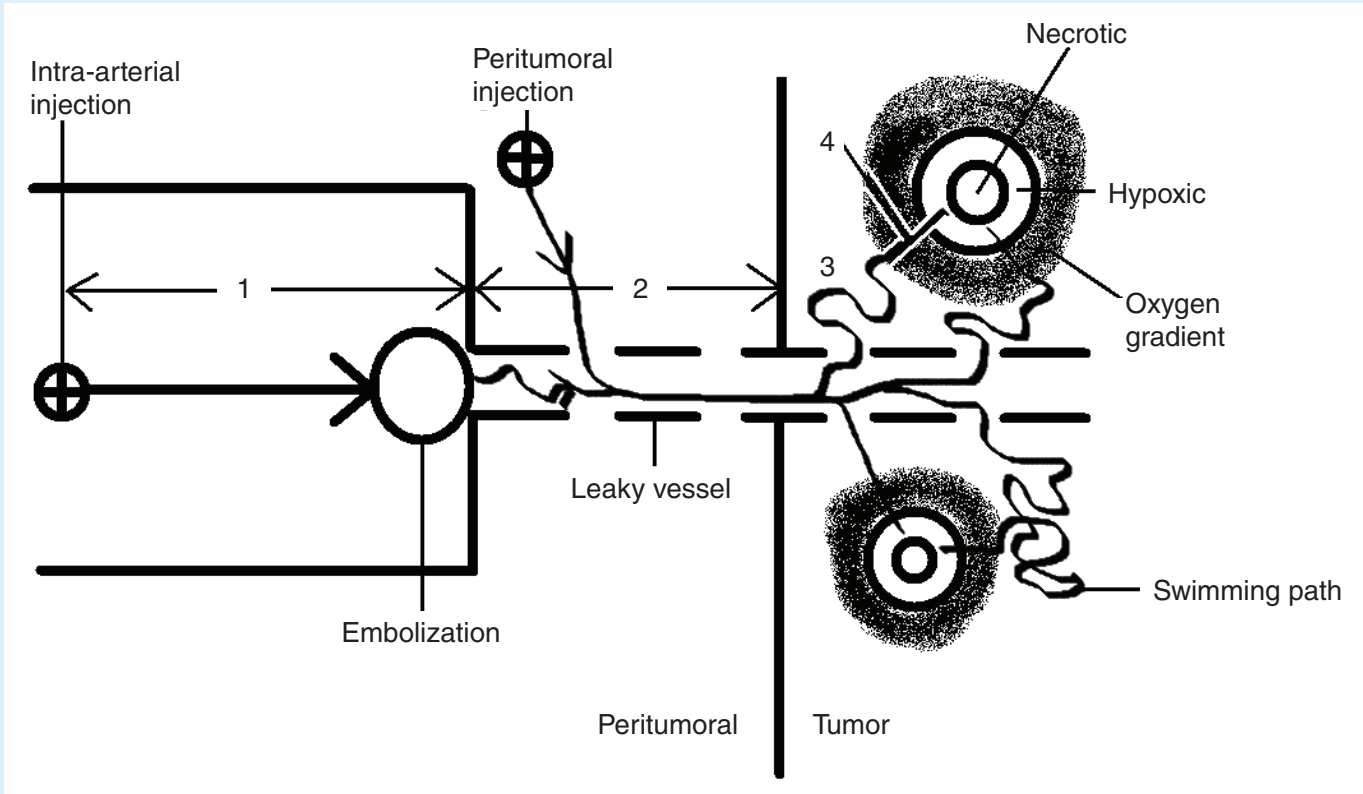

Figure 2. Simplified schematic representing the general navigation paths to target the tumor hypoxic zones from an intra-arterial or a peritumoral injection. If a peritumoral injection is possible, the loaded MC-1 cells are guided toward the tumor by a directional magnetic field (magnetotactic guidance (2) in the schematic). The loaded cells can enter and exit the leaky vessels that bring nutrients and oxygen to the tumor, hence providing entry points to travel deeper into the tumor. Once in the tumor, magnetotactic guidance is turned off and the loaded MC-1 bacteria begin a random search (3) if too far to detect an oxygen gradient from a hypoxic zone. Once the MC-1 cell detects an oxygen gradient, the swimming path follows the direction of high-to-low oxygen concentrations (4) until it detects the oxygen threshold corresponding to a region of active (duplicating) cancer cells. The MC-1 cell then maintains its position until the release of the therapeutic payloads. The detection of this oxygen threshold is critical to avoid bypassing the hypoxic zones and deliver therapeutic cargos in necrotic areas which would significantly reduce if not completely eliminate the therapeutic effects. If intra-arterial injection is more suitable, vascular navigation such as MRN and/or DFN of aggregated therapeutic-loaded MC-1 cells can be used up to an embolization site (1), releasing the loaded bacteria in the peritumoral region where their displacement capability becomes more effective.

DFN: Dipole field navigation; MRN: Magnetic resonance navigation.

Once this would be positioned inside the magnetotaxis platform (Figure 3), image registration could be automatically initiated to properly determine the position of the tumor previously gathered using the MRI scanner in this particular example. Generally, an x-ray system positioned next to the magnetotaxis platform would also be used for this purpose. To compensate for potential motion artifacts during the procedure, an option to retain maximum accuracy in targeting would be to increase the aggregation or targeting zone to an acceptable level since this would also increase the aerotactic searching area of the MC-1 cells.

Once the image registration would be completed, the therapeutic-loaded MC-1 bacteria would be injected in the peritumoral region while the computer would automatically determine and send the proper ratios of electrical currents circulating in the various coils of the magnetotaxis platform. This would be done in order to generate the magnetic fields necessary to force the therapeutic-loaded MC-1 cells to migrate toward the targeted volume preidentified by the medical specialist.

To create a single magnetic pole (monopole) corresponding to the predetermined targeted volume, known also as the aggregation zone, and which corresponds to the volume where the bacteria will aggregate using particular directional magnetic fields, special sequences generating time-multiplexed directional magnetic fields would be automatically initiated (see [16] for an example of a sequence).

During this initial magnetotactic guidance phase as represented schematically by path 2 in Figure 2, the strength of the directional magnetic field would be automatically set to induce a sufficiently strong directional torque on the chain of magnetosomes in each MC-1 cell in order to guide them toward the preidentified targeted volume or aggregation zone while being 


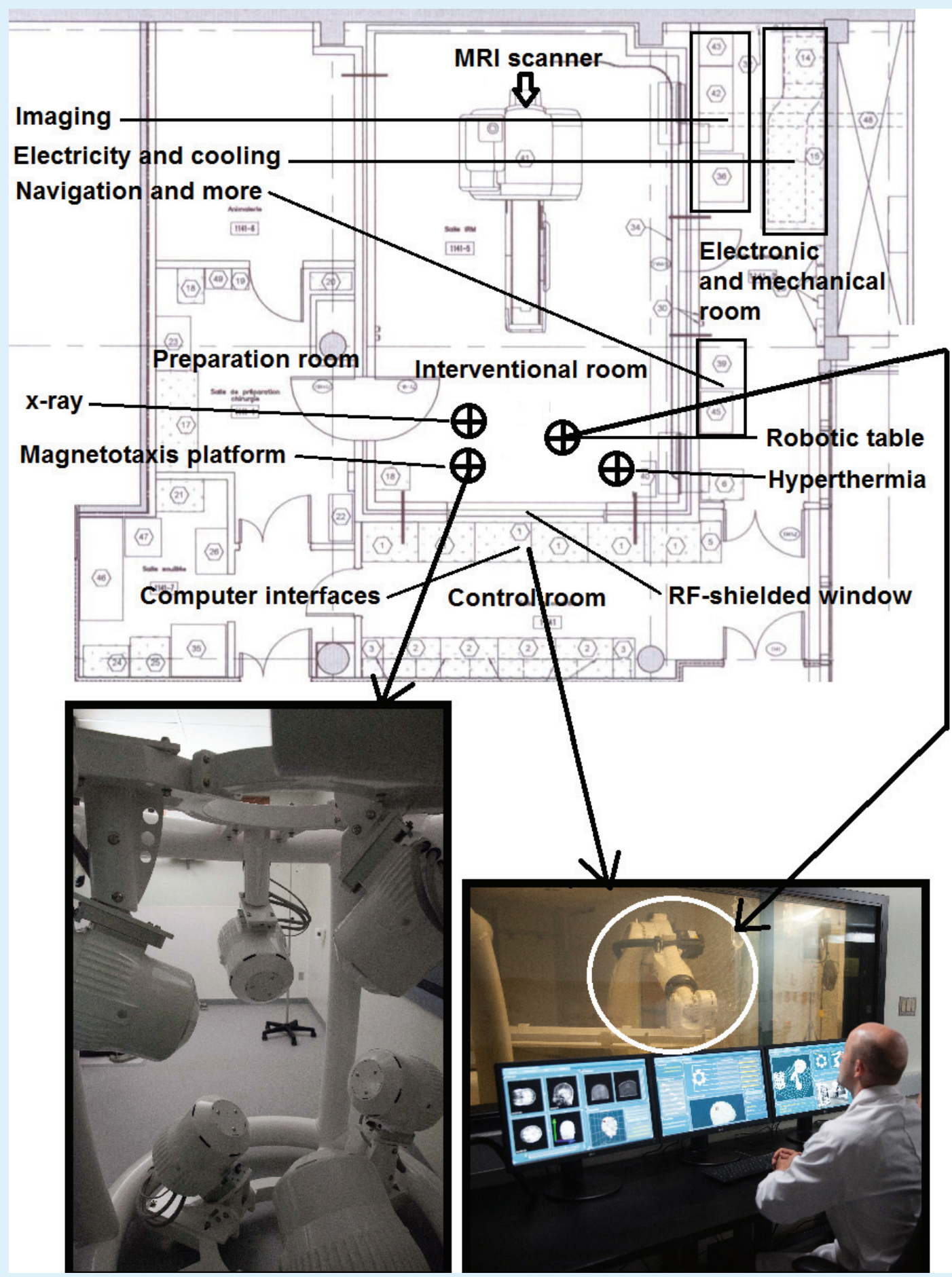

Figure 3. One example of a suitable interventional suite for maximum targeting accuracy while supporting a relatively large range of possible therapeutic modalities and target physiological sites. Here, an MRI scanner is used for imaging, localizes the tumoral region as well as other tasks such as targeting assessment of the smart delivery agents. The same MRI scanner can also be used for vascular navigation in larger blood vessels such as in arteries and larger arterioles using MRN and/or DFN. The magnetotaxis platform is used to magnetically guide the loaded MC-1 cells acting as smart delivery agents toward the tumor hypoxic zones. A hyperthermia station can also be added to temporary open the BBB if necessary. A robotic table is used to move the patient between the various platforms during the interventional procedure, which is executed with the help of special computer interfaces. Such interventional suite can be modified by removing or adding equipment such as a CT scanner or a platform for radiation therapy, to list only two examples of possible upgrades.

BBB: Blood-brain barrier; DFN: Dipole field navigation; MRN: Magnetic resonance navigation. Source: NanoRobotics Laboratory, Polytechnique Montréal, Canada. 
sufficiently low inside the targeted volume so that the aerotactic homing phase could be initiated, the latter being represented schematically by paths 3 and 4 in Figure 2. Any MC-1 cells escaping the targeted volume if swimming following a random search pattern in the absence of an oxygen gradient would be forced to return to the aggregation zone due to the induction of a directional torque by the surrounding magnetic field pointing toward the predetermined targeted tumoral region.

After a relatively short period of time that would typically last just a few minutes but no more than the expected lifetime of approximately $30 \mathrm{~min}$ of the MC-1 cells following the injection, the patient would be transferred back robotically to the MRI scanner to validate and assess the targeting efficacy provided that the MC-1 constructs would be synthesized as theranostic agents, in other words, that MRI contrast agents such as clusters of superparamagnetic iron-oxide nanoparticles would be attached with the therapeutic vectors to the surface of the MC-1 cells.

\section{Intra-arterial interventional procedure: catheterization}

If an injection in the periphery of a tumor is not clinically possible or suitable, injection in the arterial network becomes an option. From an intra-arterial injection, targeting the hypoxic regions could also be achieved with the MC-1 cells acting as therapeutic carriers with the same targeting procedure as described in the preceding section. The only difference is that since the therapeutic-loaded MC-1 bacteria would not be injected in the periphery of the tumor, they would need to be sent sufficiently near the tumor by transiting through larger vessels such as arteries and larger arterioles (path 1 in Figure 2). Because of the excessive blood flow in larger vessels and the relatively long distance separating the injection site to the tumor, injecting the bacteria directly in the arterial network without the use of an appropriate transport method would result in a much lower targeting ratio. The latter fact has been validated by our group with MC-1 cells being injected in the tail of mice bearing two tumor xenografts instead of injecting them peritumorally. Although in the former case, the target tumor contained much more bacteria than the other one, the targeting ratio was significantly inferior to those obtained with peritumoral injections, hence confirming the need to transport therapeutic-loaded MC-1 cells closer to the tumoral volume in order to achieve a much higher therapeutic index.

One approach that can be considered to initially transport the therapeutic-loaded bacteria through larger blood vessels is catheterization. The main idea is to position the tip of a catheter as close as possible to the site of the tumor by navigating it through the arterial network prior to release of the therapeutic-loaded bacteria at the tip of the catheter. This can be done by initially introducing a guidewire prior to insert the release catheter or by the use of the catheter alone. This can be done with known catheterization techniques.

But when designed to navigate deeper in narrower vessels and beyond the limit of present catheterization in order to reach closer to the peritumoral region, the diameter of the instrument (guidewire or catheter) must be reduced significantly, which results in a reduction of its stiffness. This reduced stiffness opposing the increasing friction force against the vessels walls, when traveling along tortuous vessels leading deeper in the vasculature, causes the instrument to bend before it penetrates further when being pushed. In this case, in order to go deeper and closer to the tumoral region, a pulling force must be induced at the tip of the instrument. One idea that is presently investigated is to induce a very high directional pulling magnetic gradient on the magnetic tip of the instrument. In the setup depicted in Figure 3 , such a strong pulling gradient is produced by the superconducting magnet of the clinical MRI scanner. Typically, this superconducting magnet generates a static uniform magnetic field (known as the $\mathrm{B}_{0}$ field and being at $3 \mathrm{~T}$ in the setup, shown in Figure 3 ) inside the tunnel of the scanner. Although being homogeneous inside the tunnel, such a high magnetic field decays rapidly outside and in proximity of the scanner. This fast decaying field, known as the fringe field, surrounding the MRI scanner is investigated to be used in the interventional room depicted in Figure 3 , as a very strong magnetic gradient that could pull the magnetic tip of a guidewire or catheter with a very high force. Preliminary experiments performed in phantoms confirmed the possibility of using this approach to steer catheters and guidewires deeper in the vascular network. But although being planned, no in vivo experiments have been conducted yet.

Although superconducting magnets generate the highest magnetic fields, they are better suited at producing a static magnetic field or a relatively very slow varying field, and they are bulky. The size and weight of the superconducting magnet alone prevent it to be moved in order to change the direction of the magnetic gradient. Instead, it is anticipated that the patient would be positioned and moved around the MRI scanner by the robotic table to provide a strong gradient according to the direction required to travel in each successive branch of the arterial network leading to the embolization or release site (Figure 2). This is referred to as fringe field navigation (FFN) [17]. 
In the planned scenario using the interventional suite depicted in Figure 3, besides gathering the spatial location of the tumor, an x-ray or MRI angiography with the injection of proper contrast agents would be initially done to gather an image of the arterial route separating the injection site and the release site corresponding to the embolization site depicted in Figure 2. The image of the arterial network would then be processed and exported to one of the computer displays in the control room (Figure 3). When the operator would move the position of the tip of the instrument on the computer display, the instrument would automatically be pushed and the patient would be robotically moved and oriented at a specific position within the fringe field in order to induce the proper directional pulling force on the magnetic tip. Once the FFN process would be completed, the magnetic tip would be removed, and the patient would be robotically transferred to the magnetotaxis platform prior to injecting the bacteria through the catheter once image registration would have been completed and the magnetic field to guide the bacteria would be switched on. From there, the targeting process would become the same as the one described in the previous section.

\section{Intra-arterial interventional procedure: vascular navigation}

In many instances, it is expected that the tip of the release catheter would not be positioned sufficiently close to the tumor, or the use of a catheter would not be suitable such as in the case for the need of many repetitive catheterization procedures that would be time consuming while increasing the risk of damaging the vessel walls. For such a case, efforts are presently pursued to develop a microcarrier to transport the therapeutic-loaded MC-1 cells to the periphery of the tumor by navigating them through a predetermined vascular route separating the injection point to an embolization site where the bacteria would be released (path 1 in Figure 2).

There are two main vascular navigation methods that can be used alone or in combination for this purpose. One is known as magnetic resonance navigation (MRN) [18] and the other as dipole field navigation (DFN) [19]. Unlike FFN, these two approaches provide very high field strength with fast directional changes, two characteristics that are highly desirable when navigating therapeutic agents or carriers in the arterial network. The three navigation methods are depicted in Figure 4. Briefly, for FFN the patient would be placed outside the MRI scanner, and the directional changes would be done by robotically changing the position of the patient in the external fringe field generated by the superconducting magnet of the MRI scanner being represented by positions one and two in Figure 4. For MRN, the patient would be positioned inside the tunnel of the MRI scanner, and directional changes during navigation would be done by varying the directions of the gradients generated by the imaging gradient coils of the scanner being represented by the larger arrows in Figure 4. MRN has been used for the first time to navigate a microscopic bead in the hepatic artery of a swine [18] and later for the delivery of therapeutics in specific regions in the liver of animal models following navigation in the hepatic artery. Presently, medical protocols are being developed to exploit MRN for potential future interventions in humans.

For DFN, the patient would also be positioned inside the tunnel of the MRI scanner but relatively large magnetic cores, represented as circles inside the tunnel in Figure 4, would be positioned around the patient to distort the high static field of the scanner. From experiments done in phantoms, we already know that these distortions create very high directional gradients that force the therapeutic agents to follow a predetermined path. The positions of the large cores are determined using a special algorithm that has been successfully tested and which is executed on a computer program based on the preacquired images of the vascular network that must be traveled.

Confirmed in previous in vivo experiments, magnetic untethered therapeutic carriers are typically navigated too deep in the vascular network to allow recovery unlike for the magnetic tip of a guidewire or a catheter. As such, superparamagnetic nanoparticles are typically considered because of the lack of residual magnetization, which prevents the potential formation of unwanted large clusters once the patient would be removed from the magnetic field used during the vascular navigation phase. As such, sufficient field strength must be provided during the navigation phase to bring these superparamagnetic nanoparticles at or as close as possible to saturation magnetization in order to induce the maximum directional displacement force from a given magnetic gradient. Although the magnetic gradient is much higher for FFN, the field strength is lower than for MRN and DFN, which is not a concern for a permanent magnetic tip but a real constraint for superparamagnetic nanoparticles. But another issue with FFN is the rate at which the direction of the magnetic gradient can be varied during navigation. Although the relatively slow directional change performed by the displacement of the robotic table proved to be adequate during catheterization, the navigation of untethered magnetic microcarriers such as the ones transporting therapeuticloaded MC-1 bacteria would typically require much faster directional changes.

Upon completion of the vascular navigation phase, 


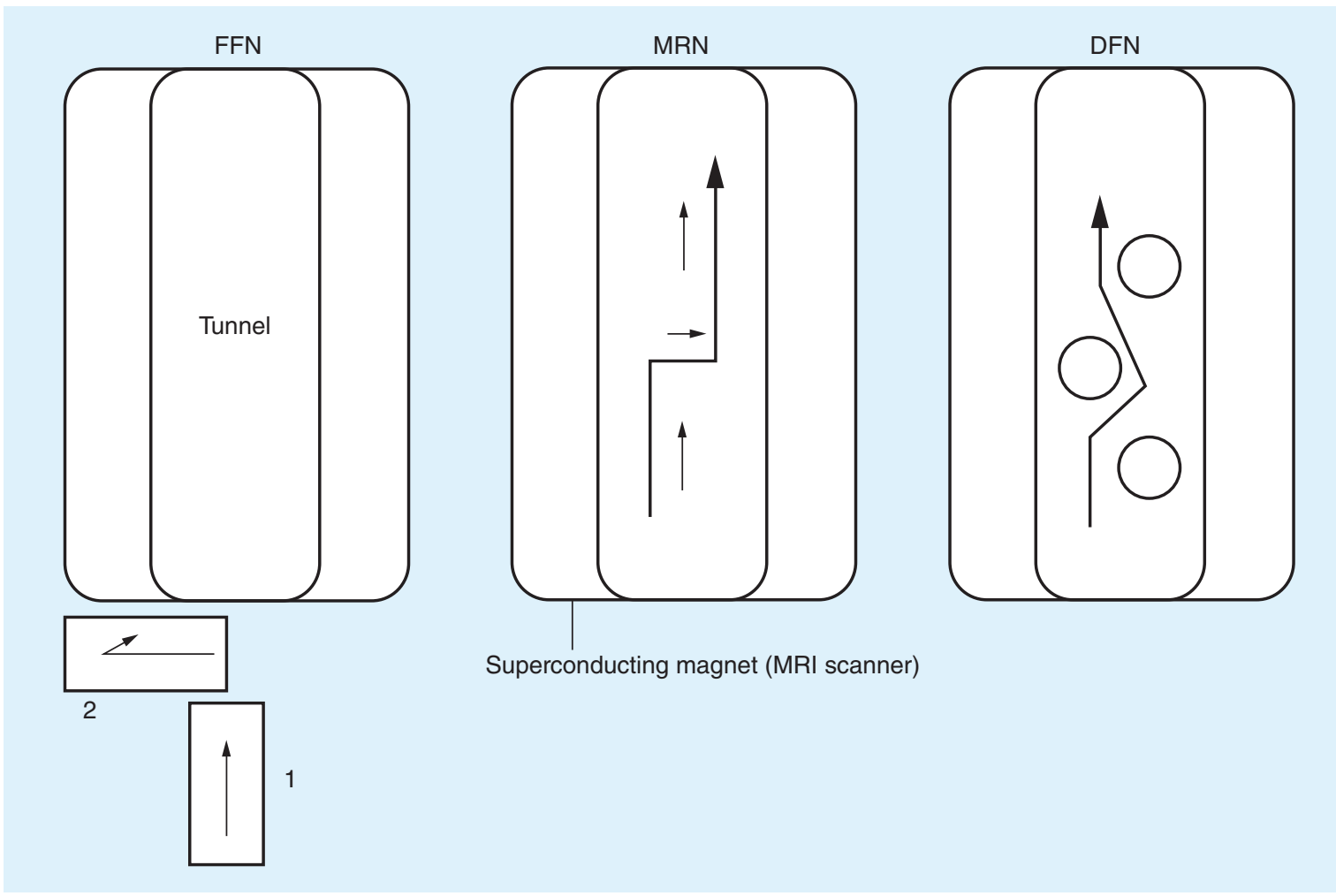

Figure 4. Simple schematics representing the three main vascular navigation methods: fringe field navigation, magnetic resonance navigation and dipole field navigation. One or a combination of these methods can be used to transport therapeutic-loaded MC-1 bacteria closer to the tumoral region from the position of the intra-arterial injection.

the position of the embolization or release site would typically be validated through MRI sequences and displayed in the control room (Figure 3). Both the navigation phases between the intra-arterial injection and the embolization sites, and the transfer of the patient from the MRI scanner to the magnetotaxis platform (see Figure 3), must be done as quickly as possible. The reason is that the motility of the therapeutic-loaded MC-1 bacteria gradually decreases once injected in the body, and initial studies suggest that most of them will be nonmotile after $30 \mathrm{~min}$. Transferring the patient using a computer-controlled robotic table prior to automatic registration and generation of the appropriate magnetic fields toward the tumor allow them to shorten the overall procedure time in order to take advantage of the high motility of the bacterial carriers.

\section{Crossing the blood-brain barrier}

The same superparamagnetic nanoparticles that can be used for inducing a force on therapeutic agents during vascular navigation and to act as MRI contrast agents for detecting the location of these navigable agents including MC-1 bacteria, when clusters of such superparamagnetic nanoparticles are attached to the surface of the cells, can also be synthesized with characteristics that will persuade the diffusion of therapeutic molecules through the blood-brain barrier (BBB) [20]. Using such clusters of nanoparticles carried by navigable agents such as the MC-1 bacteria, for instance, a local transient disruption of the BBB prior to a 2-h recovery period can be achieved via hyperthermia from the superparamagnetic nanoparticles when exposed to an alternating magnetic field generated by a hyperthermia station (see Figure 3).

Again, transporting these magnetic nanoparticles alone, allowing also for hyperthermia-based treatments and MRI contrast enhancement or in combination with other therapeutic agents could be achieved using only one or a combination of the preceding delivery methods. Once, at the target location in the brain, the patient could be robotically moved from the magnetotaxis platform to the MRI scanner to confirm the location of the MC-1 cells, moved from the fringe field to inside the tunnel of the MRI scanner if FFN was used alone or remain in the MRI scanner if MRN or DFN was used without MC-1 bacteria. Once the position of the clusters of magnetic nanoparticles would have been assessed and confirmed, the patient could then be robotically moved to the hyperthermia station. Once at the hyperthermia station, no image registration would be required since variations in tissue densities do not affect the path of magnetic fields 
unlike the sound waves used in high-intensity focus ultrasounds [21], although the latter approach could also be used with the proposed nonsystemic delivery methods. One potential particular advantage of this approach compared with high-intensity focus ultrasounds is that the cellular level hyperthermia process is done at the exact location where the clusters of nanoparticles and the therapeutic molecules have been delivered by the same navigable agents. Therefore, when positioned inside the hyperthermia station, the generated alternating magnetic field results in a local elevation of the temperature that increases temporarily the permeability of the $\mathrm{BBB}$ at the exact location where the therapeutics have been delivered and will not be affected by motions of the head during the interventions. Nonetheless, although initial experiments performed in the brain of small animals are encouraging, more in vivo tests are presently pursued to validate its potential usage for humans.

\section{Conclusion}

When the location of the target can be spatially determined as it is generally the case for solid tumors or specific metastatic cancers, therapeutic delivery can take advantages of navigable agents [22] and, particularly, new smart delivery agents with the functionalities and characteristics required to directly target active cancer cells. In such a case, passively drifting therapeutics resulting in a low therapeutic index could become much more effective at targeting regions that would lead to optimal therapeutic outcomes by taking advantages of the propulsion, navigation and homing capabilities provided by these smart delivery agents. Presently, these smart delivery agents are in the form of MC-1 magnetotactic bacteria being harnessed to act as medical nanorobots or 'smart bullets.' If injection must be performed outside the magnetoaerotactic targeting range of these bacteria, in other words, beyond the peritumoral regions, then they must be transported closer to the tumor. This could be done using magnetic-based catheterization relying on a new method-dubbed FFN that would allow the release of these therapeutic-loaded bacteria deeper in the vasculature; by navigating them in larger microcarriers along a predetermined path in the vascular network using techniques such as MRN or DFN; or using a combination of these methods.

Presently, all the tools, platforms and methods exist to target and deliver therapeutics at a specific location in the human body including the brain. New medical interventional protocols taking advantages of these new technologies are being developed. But one of the major obstacles remains the openness to such new approaches and technologies since those represent a radical shift from the more traditional delivery methods relying on systemically circulating therapeutic agents. If these 'smart' delivery vehicles, capable of transporting a multitude of therapeutic agents, could be adopted and proved to be safe for humans (note: safety tests for the MC-1 cells have already been done in rats and mice, and the results are very encouraging), then the efficacy of a given therapeutic vector could be significantly enhanced using a much smaller dose leading to minimum systemic toxicity for the patients.

\section{Future perspective}

So far, complementing therapeutic deliveries with the appropriate robotic functionalities, made possible by applying engineering principles, has already shown far superior targeting efficacy in regions of active cancer cells in animal models well beyond the diffusion limit of passively drifting therapeutic vectors. Then, logically, it will be no surprise to witness in the near future the therapeutic efficacy of therapeutics being significantly improved by the delivery of these vectors in regions allowing maximum therapeutic effects. Going further, robotically assisted delivery as a new and complementary alternative in improving the therapeutic efficacy besides the synthesis of new molecular constructs and functionalization approaches alone will most likely be a more popular option in the future. Such an approach could also be considered for the synthesis of enhanced therapeutic vectors from generic drugs, providing a second life to the latter.

It is also anticipated that at some point in the future, such 'smart' delivery approaches could be applied to a multitude of therapeutic modalities even for immunotherapy since tumor hypoxia is a concern since it can reduce not only the effectiveness of conventional therapies but also cancer immunotherapy. In this context, critical hypoxia-associated pathways are attractive targets for immunotherapy of cancer. Therefore, the methods proposed here may support the development of more durable and successful cancer immunotherapy approaches in the future as well.

Another issue often occurring in the treatment of metastatic cancers is that after a relatively long treatment phase, the drug stops working. With a higher concentration of drug molecules delivered by these smart agents, yielding a much higher therapeutic index, combined with the fact that such delivery is done well beyond the diffusion limits and directly to the regions of active cancer cells, the therapeutic efficacy could potentially increase by several orders of magnitude. As such, the length of the treatment could potentially be reduced significantly which may help preventing the drug to stop working during treatments. Furthermore, with the capability 
to target stem cells, the proposed methods could potentially eliminate the recurrence of the metastatic lesions as well. Another interesting fact is that since the aerotactic displacement of the MC- 1 bacteria is entirely autonomous, invisible or undetected metastatic tumors can also be targeted by the bacterial carriers, hence providing a more powerful therapeutic modality that is not affected by limitation in the spatial resolution and detection capabilities of existing medical imaging systems. All these facts will most likely motivate its investigation for specific metastatic cancers within the next few years.

But some more steps are expected to be pursued in the near future to bring such unconventional delivery methods to the clinics. Translational efforts to the clinics have already been initiated, and a clinically viable interventional suite capable of exploiting these 'smart' delivery agents has been scaled up successfully and put in place to allow the development of appropriate interventional protocols and computer interfaces. All these tasks including more work in platform integration and synchronization, which are presently considered as relatively low development risk efforts, will likely require up to some additional years before being entirely mature and optimal for clinical uses.

A major concern is related to safety issues and specifically the potential uses of the MC-1 bacteria in the human body. Although the MC-1 cells have shown no significant immune response and further results obtained in mice and rat models suggest that this bacterial carrier is likely to be safe, more tests likely performed on primates will be required and potentially done within the next few years. If successful, this could soon be followed by regulatory approvals to conduct the first human trials, while genetic, initially targeted at the chain of magnetosomes of magnetotactic bacteria for future developments in synthetic biology, will likely begin to play a greater role for the future development of microorganism-based therapeutic carriers.

\section{Financial \& competing interests disclosure}

Polytechnique Montréal, Univalor, Consortium Québécois sur la Découverte du Médicaments, Canada Research Chair Program, Natural Sciences and Engineering Research Council of Canada, Government of Québec, Mitacs, Canadian Foundation for Innovation, National Institute of Health, and several other organizations contributed financially to the author's research, and associated collaborators and students. The author has no other relevant affiliations or financial involvement with any organization or entity with a financial interest in or financial conflict with the subject matter or materials discussed in the manuscript apart from those disclosed.

No writing assistance was utilized in the production of this manuscript.

\section{Open access}

This work is licensed under the Attribution-NonCommercialNoDerivatives 4.0 Unported License. To view a copy of this license, visit http://creativecommons.org/licenses/by-nc$\mathrm{nd} / 4.0 /$

\section{Upgrading therapeutics with robotic functionalities to enhance delivery \& targeting}

- Engineering principles can transform systematically circulating vectors that so far were based primarily on physical characteristics and biochemical principles alone, as smart therapeutic agents with the required propulsion-navigation-homing capabilities to enable them to go straight to their intended targets.

- Unlike other delivery approaches investigated so far, such smart delivery vectors allow nonsystemic delivery and targeting of active cancer cells well beyond the diffusion limit of therapeutic agents alone.

MC-1 magnetoaerotactic bacterial carriers

- Live MC-1 bacterial cells when operating in a computer-controlled magnetic field environment can be transformed as smart delivery agents with the robotic functionalities required to target active cancer cells deep in tumoral lesions, somehow mimicking the 'magic bullet' concept. Recent experiments showed that $55 \%$ of the drug-loaded bacterial cells targeted regions of active cancer cells in tumors implanted in animal models.

- These bacteria can be used to transport a variety of therapeutics and cargos.

\section{Beyond peritumoral injection}

- Live MC-1 bacterial carriers are more effective in the physiological microenvironments in the vicinity and inside a tumor. When the injection of these smart delivery agents cannot be done peritumorally, specific methods, known as fringe field navigation, magnetic resonance navigation or dipole field navigation exploiting the magnetic environment provided by a clinical MRI scanner, can be used to navigate clusters of these MC-1 cells closer to the regions to be treated.

Translational phase

- The development of medical interventional protocols has been initiated and a first interventional suite to translate such new technologies to the clinics has been implemented. 


\section{References}

Papers of special note have been highlighted as:

- of interest; $\bullet \bullet$ of considerable interest

1 Strebhardt K, Ullrich A. Paul Ehrlich's magic bullet concept: 100 years of progress. Nat. Rev. Cancer 8(6), 473-480 (2008).

-• Review on the progress of the magic bullet concept.

2 Heldin $\mathrm{CH}$, Rubin K, Pietras K, Östman A. High interstitial fluid pressure - an obstacle in cancer therapy. Nat. Rev. Cancer 4(10), 806-813 (2004).

3 Martel S. Navigation control of micro-agents in the vascular network. IEEE Control Systems 33(6), 199-134 (2013).

4 Brown JM, Wilson WR. Exploiting tumour hypoxia in cancer treatment. Nat. Rev. Cancer 4(6), 437-446 (2004).

- Review explaining the importance of targeting hypoxic zones in cancer treatment.

5 Vaupel P. The role of hypoxia-induced factors in tumor progression. Oncologist 9 (5), 10-17 (2004).

6 Hashizume H, Baluk P, Morikawa S et al. Openings between defective endothelial cells explains tumor vessel leakiness. Am. J. Pathol. 156(4), 1363-1380 (2000).

7 Martel S. Bacterial microsystems and microrobots. Biomed. Microdevices 14(6), 1033-1045 (2013).

8 Blakemore RP. Magnetotactic bacteria. Science 190(4212), 377-379 (1975).

- Good introduction to magnetotactic bacteria.

9 Bazylinski DA, Williams TJ, Lefèvre CT et al. Magnetococcus marinus gen. nov., sp. nov., a marine, magnetotactic bacterium that represents a novel lineage (Magnetococcaceae fam. nov., Magnetococcales ord. nov.) at the base of the Alphaproteobacteria. Int. J. Syst. Evol. Microbiol. 63(3), 801-808 (2013).

10 Felfoul O, Mohammadi M, Taherkhani S et al. Magnetoaerotactic bacteria deliver drug-containing nanoliposomes to tumour hypoxic regions. Nat. Nanotechnol. 11(11), 941-947 (2016).

- Showing the targeting efficacy of drug-loaded MC-1 bacteria in tumor hypoxic zones.

11 Lefèvre CT, Bennet M, Landau L et al. Diversity of magneto-aerotactic behaviors and oxygen sensing mechanisms in cultured magnetotactic bacteria. Biophys. J. 107(2), 527-538 (2014).

12 Groebe K, Vaupel P. Evaluation of oxygen diffusion distances in human breast cancer xenografts using tumor-specific in vivo data: role of various mechanisms in the development of tumor hypoxia. Int. J. Radiation Oncol. Biol. Phys. 15(3), 691-697 (1988).
13 Schüler D. Formation of magnetosomes in magnetotactic bacteria. J. Mol. Microbiol. Biotechnol. 1(1), 79-86 (1999).

14 McKeown SR. Defining normoxia, physoxia and hypoxia in tumours - implications for treatment response. Br. J. Radiol. 87, 20130676 (2014).

15 Taherkhani S, Mohammadi M, Daoud J, Martel S, Tabrizian $\mathrm{M}$. Covalent binding of nanoliposomes to the surface of magnetotactic bacteria acting as self-propelled target delivery agents. ACS Nano 8(5), 5049-5060 (2014).

-. Describing the attachment process to the MC-1 bacterial carriers.

16 de Lanauze D, Felfoul O, Turcot JP, Mohammadi M, Martel $\mathrm{S}$. Three-dimensional remote aggregation and steering of magnetotactic bacteria microrobots for drug delivery applications. Int. J. Robot. Res. 33(3), 359-374 (2014).

- Showing an example of magnetic field sequences used to aggregate MC-1 bacteria in a $3 \mathrm{D}$ location.

17 Tremblay C, Conan B, Loghin D, Bigot A, Martel S. Fringe field navigation for catheterization. Presented at: European Conference of the International Federation for Medical and Biological Engineering. Dubrovnik, Croatia, 379-382 (2014).

18 Martel S, Mathieu JB, Felfoul O et al. Automatic navigation of an untethered device in the artery of a living animal using a conventional clinical magnetic resonance imaging system. Appl. Phys. Lett. 90(11), 114105 (2007).

•- First in vivo demonstration of magnetic resonance navigation.

19 Latulippe M, Martel S. Dipole field navigation: theory and proof of concept. IEEE Trans. Robotics 31(6), 1353-1363 (2015).

-• Good introduction to dipole field navigation.

20 Tabatabaei SN, Girouard H, Carret AS, Martel S. Remote control of the permeability of the blood-brain barrier by magnetic heating of nanoparticles: a proof of concept for brain drug delivery. J. Control. Release 206, 49-57 (2015).

-• Describing the fundamental principles of opening the blood-brain barrier with local hyperthermia of nanoparticles.

21 Kennedy JE. High-intensity focused ultrasound in the treatment of solid tumours. Nat. Rev. Cancer 5(4), 321-327 (2005).

22 Martel S. Magnetic therapeutic delivery using navigable agents. Ther. Deliv. 5(2), 1-16 (2014).

- Providing further details on the various approaches to navigate therapeutic agents. 